\title{
Validation and optimization of a predictive model for radiation pneumonitis in patients with lung cancer
}

\author{
CHARLOTT MÖRTH $^{1,2}$, IOANNIS KAFANTARIS ${ }^{3}$, MARKUS CASTEGREN $^{1,4}$ and ANTONIOS VALACHIS ${ }^{1,2}$ \\ ${ }^{1}$ Centre for Clinical Research Sörmland, Uppsala University; Departments of ${ }^{2}$ Oncology and ${ }^{3}$ Pulmonary Medicine, \\ Mälarsjukhuset, SE-631 88 Eskilstuna; ${ }^{4}$ Department of Anesthesia, Surgical Services and Intensive Care, \\ Karolinska University Hospital Huddinge, Karolinska Institute, SE-171 76 Stockholm, Sweden
}

Received January 22, 2016; Accepted April 15, 2016

DOI: $10.3892 / \mathrm{ol} .2016 .4678$

\begin{abstract}
The aim of the current retrospective study was to validate a predictive model for radiation pneumonitis (STRIPE) in an independent dataset and to investigate whether the addition of other potential risk factors could strengthen the accuracy of the model. Consecutive patients with non-small cell lung carcinoma (NSCLC; $n=71$ ) treated with definitive concurrent chemotherapy and radiotherapy were retrospectively assessed for radiation pneumonitis (RP). The results identified that 16 (23\%) patients developed grade $\geq 2$ RP. Furthermore, STRIPE score (intermediate vs. low risk) was independently associated with the development of RP [odds ratio (OR), 3.72; 95\% confidence interval (CI), 1.00-13.89], whereas current smoking status was found to be protective against RP (OR, $0.09 ; 95 \%$ CI, 0.01-0.78). Similar discriminatory power of the STRIPE score was observed as in the original study. The addition of smoking status strengthened the model's discriminatory ability to predict RP. Thus, the addition of smoking status as a risk factor may strengthen the accuracy of the model for predicting RP in patients with NSCLC.
\end{abstract}

\section{Introduction}

Lung cancer represents the leading cause of cancer-related mortality worldwide and among newly diagnosed cases, with non-small cell lung cancer (NSCLC) accounting for $~ 80 \%$ of cases (1).

Surgery remains the primary curative treatment strategy in patients with early-stage NSCLC, however, its role in patients with locally advanced NSCLC is controversial (2). For these patients, concurrent chemoradiation therapy (CCRT) is considered the standard of care, considering the benefit of CCRT

Correspondence to: Dr Charlott Mörth, Department of Oncology, Mälarsjukhuset, 34 Kungsvägen, SE-631 88 Eskilstuna, Sweden E-mail: charlott.morth@dll.se

Key words: non-small cell lung cancer, radiation pneumonitis, radiotherapy, concurrent chemoradiation therapy on survival compared with radiotherapy alone or sequential chemotherapy and radiation therapy (3-5).

However, the major disadvantage of CCRT is the risk of developing radiation pneumonitis (RP). In particular, RP results in the development of aseptic inflammation of the lung, which causes dry cough, dyspnea, fever and, in the most severe cases, respiratory failure and mortality (6-8). The incidence of all-grade RP following CCRT ranges between 13 and $37 \%$, whereas the incidence of severe RP (grades III-IV) has been reported in $\leq 20 \%$ of cases (6-8). Considering the substantial negative impact of RP on quality of life and prognosis (9), it is essential to stratify patients according to their risk of developing RP prior to the initiation of CCRT, in order to individualize the treatment strategy.

Several efforts have been made to identify patient-, tumor- and dose-related factors that may influence the risk of RP (9-11). However, the proposed predictive models have limited clinical utility, predominantly due to the lack of validation of the models in an independent dataset $(9,10)$, and the omission of important clinical and treatment-related confounding factors (11). Recently, an individual patient meta-analysis presented a predictive model for RP (STRIPE project) that divided the patients into three different risk groups based on the type of chemotherapy used, the age of the patient and two dosimetric parameters [the mean lung dose (MLD) and V20] (12). The major limitation of the current meta-analysis was the exclusion from the final model of certain variables that have been previously reported as predictors of RP, such as smoking status, comorbidities and pulmonary function (11), due to the lack of adequate data.

The aim of the current retrospective study was to validate the predictive model proposed by the previous individual patient meta-analysis in an independent dataset. In addition, the study aimed to investigate whether the addition of other potential risk factors could strengthen the accuracy of the model.

\section{Materials and methods}

Study design and setting. The present bi-institutional, retrospective, cohort study included patients with locally-advanced, inoperable NSCLC treated with definitive CCRT. The two institutions that contributed were the Department of Oncology, 
Mälarsjukhuset (Eskilstuna, Sweden) and the Department of Pulmonary Medicine, Västerås Central Hospital (Västerås, Sweden).

Patient population. The electronic medical records of each hospital were searched to identify all patients with locally-advanced, inoperable NSCLC that received definitive CCRT between January 2008 and December 2012. In addition to the hospital medical records, the lung cancer tumor registry (The Regional Tumor Quality Registry of UppsalaÖrebro counties; Uppsala, Sweden) was also used to identify patients planned for definitive CCRT. Patients with concurrent distant metastases and those who did not receive CCRT were excluded. In total, 71 patients were considered eligible for the study.

The study was approved by the local review board (EPN no. 2013/590-31/1; Regional Ethical Review Board in Stockholm, Stockholm, Sweden), which concluded that there was no need for informed consent due to the retrospective nature of the study.

Radiation therapy. Radiation therapy was delivered once daily with external beam radiotherapy using a three-dimensional conformal technique. Planning computed tomography (CT; in Eskilstuna, Light Speed Pro16RT; GE Healthcare Life Sciences, Chalfont, UK; in Västerås, Brilliance CT Big Bore; Philips Medical Systems, Inc., Bothell, WA, USA) was required to determine target volumes. The gross tumor volume (GTV) included the primary tumor volume and positive mediastinal lymph nodes, as defined on CT imaging. The clinical target volume (CTV) included the GTV plus a margin of $1-1.5 \mathrm{~cm}$. The planning target volume (PTV) was defined as the CTV with the addition of a margin of $0.5-1 \mathrm{~cm}$. The prescribed doses of radiotherapy were 2 Gy per fraction daily (Monday-Friday) with a total dose range of 46-68 Gy. The MLD (MLD delivered to the total lung volume minus the PTV) and V20 (percentage of CT-defined total lung volume receiving a radiation dose of $\geq 20 \mathrm{~Gy}$ ) were used as dosimetric parameters to represent the lung volumes irradiated.

Chemotherapy. The concurrent chemotherapy was either monotherapy $\left[60 \mathrm{mg} / \mathrm{m}^{2}\right.$ paclitaxel intravenous (i.v.) per week, administered over $60 \mathrm{~min}$ once per week] or platinum-based chemotherapy $\left[80 \mathrm{mg} / \mathrm{m}^{2}\right.$ i.v. cisplatin or area under the curve (AUC) 5 i.v. carboplatin administered over $60 \mathrm{~min}$ on day $1 ; 25 \mathrm{mg} / \mathrm{m}^{2}$ i.v. vinorelbine administered over $15 \mathrm{~min}$ or $60 \mathrm{mg} / \mathrm{m}^{2}$ oral vinorelbine on days 1 and 8 every 21 days]. All treatments included $8 \mathrm{mg}$ betamethasone i.v. on day 1. Certain patients were administered continuous treatment with corticosteroids due to their symptoms. The majority of the patients (70/71 patients) received platinum-doublet induction chemotherapy for 2-4 courses, according to the Swedish National guidelines (www.cancercentrum.se/uppsala-orebro/cancerdiagnoser/lunga-och-lungsack/vardprogram). The drugs were supplied by the pharmacy, and so the choice of supplier was subject to change according to the pricing and local agreements.

Data collection. The medical records and follow-up data of the patients were retrospectively extracted. The following data were recorded in a pre-specified database: Gender, age, smoking status, comorbidities, tumor location, tumor size, tumor stage (13), histology, patient performance status (according to the Eastern Cooperative Oncology Group score at the start of treatment) (14), weight loss (>10\% weight loss during the 6 months prior to diagnosis), total radiotherapy dose, MLD, V20, PTV and type of concurrent chemotherapy received. In addition, the STRIPE score (12) was retrospectively calculated for each patient.

Outcomes. The primary endpoint of the study was the development of RP. The severity of RP was assessed according to the Radiation Therapy Oncology Group scale (grade 0-5) (15) based on described symptoms, clinical judgments and radiation findings.

During the course of radiation therapy, radiation oncologists evaluated the patients regularly to identify early signs of RP. A clinical evaluation with staging was repeated every 2-3 months after completion of treatment using contrast-enhanced CT. The medical records were used by two investigators (C.M and I.K) to identify patients with RP and the severity grade. In case of discrepancy between the initial grading of RP and the investigator's assessment, a consensus was made by discussion between the investigators.

Statistical analysis. Categorical variables are expressed as $\mathrm{n}(\%)$ and continuous variables as median (range). The potential risk factors for grade $\geq 2 \mathrm{RP}$ were compared using Student's t-test or the Mann-Whitney U test (in case of non-normally distributed variables) for continuous variables and the $\chi^{2}$ test or Fisher's exact test, when applicable, for categorical variables. Any variables significantly associated with the development of grade $\geq 2$ pneumonitis on bivariate analysis (with a cut-off value of $\mathrm{P}<0.05$ ) were considered for entry into a multivariate stepwise logistic regression analysis to optimize it. To identify the impact of each variable in the optimized model, the AUC was re-calculated for each variable included in the model.

To address the secondary aim of the current study (validation of the STRIPE score), the discriminatory power and calibration of the STRIPE score was calculated in the patient population. Due to the lack of high-risk patients in the current cohort, the validation of the STRIPE score could only be investigated to distinguish low-risk versus intermediate risk patients. The discriminatory power of the STRIPE score was evaluated by using the AUC (c-statistic) and its 95\% confidence interval (CI). An AUC of 0.5 indicates that the model has a predictive discrimination no better than chance, whereas an AUC of 1.0 indicates a perfectly discriminating model. The calibration of the STRIPE score was evaluated using the Hosmer-Lemeshow goodness-of-fit statistic, where a high $\mathrm{P}$-value indicates good calibration.

Statistical analyses were performed using SPSS software (version 20.0; SPSS, Inc., Chicago, IL, USA) and 2-sided $\mathrm{P}<0.05$ indicated a statistically significant difference.

\section{Results}

The present study identified 71 patients that received CCRT due to NSCLC and were eligible for inclusion in the study. Patient demographics, clinical and treatment characteristics, 
Table I. Demographic, clinical and treatment characteristics of eligible patients.

\begin{tabular}{|c|c|}
\hline Characteristic & Patients \\
\hline \multicolumn{2}{|l|}{ Gender $^{\mathrm{a}}$} \\
\hline Male & $33(46)$ \\
\hline Female & $38(54)$ \\
\hline Age, years ${ }^{b}$ & $71(43-78)$ \\
\hline \multicolumn{2}{|l|}{ Smoking $^{\mathrm{a}}$} \\
\hline Never & $8(11)$ \\
\hline Former & $37(52)$ \\
\hline Current & $26(37)$ \\
\hline \multicolumn{2}{|l|}{ Comorbidities $^{\mathrm{a}}$} \\
\hline Cardiac & $18(25)$ \\
\hline Diabetes mellitus & $8(11)$ \\
\hline \multicolumn{2}{|l|}{ Location of tumor ${ }^{\mathrm{a}}$} \\
\hline Upper & $29(41)$ \\
\hline Lower & $36(51)$ \\
\hline Right & $38(54)$ \\
\hline Left & $31(44)$ \\
\hline Tumor size, $\mathrm{cm}^{\mathrm{b}}$ & $4.5(1.8-11.8)$ \\
\hline \multicolumn{2}{|l|}{ Tumor stage ${ }^{\mathrm{a}}$} \\
\hline IIA & $2(3)$ \\
\hline IIB & $2(3)$ \\
\hline IIIA & $27(38)$ \\
\hline IIIB & $38(54)$ \\
\hline \multicolumn{2}{|l|}{ Histology $\mathrm{y}^{\mathrm{a}}$} \\
\hline Squamous & $26(37)$ \\
\hline Non-squamous & $39(55)$ \\
\hline \multicolumn{2}{|c|}{ ECOG PS (at the start of treatment) ${ }^{\mathrm{a}}$} \\
\hline 0 & $28(39)$ \\
\hline 1 & $32(45)$ \\
\hline 2 & $11(16)$ \\
\hline RT dose, Gy & $66(46-68)$ \\
\hline \multicolumn{2}{|c|}{ Type of chemotherapy during $\mathrm{RT}^{\mathrm{a}}$} \\
\hline Paclitaxel & $62(87)$ \\
\hline Carboplatin/vinorelbin & $6(9)$ \\
\hline Cisplatin/vinorelbin & $3(4)$ \\
\hline MLD, Gy ${ }^{b}$ & $14.0(1.4-28.0)$ \\
\hline $\mathrm{V} 20, \%^{\mathrm{b}}$ & $24(0-50)$ \\
\hline $\mathrm{PTV}, \mathrm{cm}^{\mathrm{b}}$ & $499(157-2,202)$ \\
\hline \multicolumn{2}{|l|}{ STRIPE score ${ }^{\mathrm{a}}$} \\
\hline Low risk & $37(52)$ \\
\hline Intermediate risk & $34(48)$ \\
\hline High risk & $0(0)$ \\
\hline
\end{tabular}

Data are presented as ${ }^{\mathrm{a}} \mathrm{n}(\%)$ or ${ }^{\mathrm{b}}$ median (range). ECOG PS, Eastern Cooperative Oncology Group performance status; RT, radiotherapy; MLD, mean lung dose; PTV, planning target volume.

and STRIPE scores are indicated in Table I. No patients were at a high risk of developing RP according to the STRIPE score (12), as concurrent treatment with carboplatin-paclitaxel was not used in the present patient cohort. However, 16 patients (23\%) developed grade $\geq 2$ RP. The median time from treatment to development of RP was 6 weeks (range, 0-16 weeks) (data not shown).

Bivariate analysis, as shown in Table II, revealed four variables that are potentially associated with the development of for RP $\geq 2$ : MLD, V20\%, STRIPE score and smoking status. An ongoing smoking status with current smoking appeared to be protective against RP.

Multivariate regression analysis included the STRIPE score and the smoking status, however, the MLD and the V20 were excluded, as these variables had already been included in the STRIPE score. The results revealed that the STRIPE score [intermediate vs. low risk; odds ratio (OR), 3.72; 95\% CI, 1.00-13.89] was independently associated with the development of RP, whereas current smoking was found to be protective against RP (OR, 0.09; 95\% CI, 0.01-0.78) (data not shown).

To address the secondary aim of the present study (external validation of the STRIPE score), the discriminatory power of the STRIPE score was calculated. Due to the lack of high-risk patients in our cohort, only the validation of the STRIPE score could be investigated to distinguish low-risk versus intermediate risk patients. A similar discriminatory power of the STRIPE score was observed as in the original study (AUC 0.68 in the current cohort vs. 0.66 in the original cohort) (12). The addition of smoking status to the model strengthened its discriminatory ability to predict RP (AUC 0.72 vs. 0.68 for STRIPE score only) (Table III).

\section{Discussion}

The present study describes the first attempt to validate and, if possible, optimize the STRIPE score in an independent cohort of patients with NSCLC. The results revealed that the STRIPE score had a comparable discriminatory power in the current cohort as in the original cohort (12). The current study also showed that the addition of smoking status as a risk factor for RP could strengthen the accuracy of the model.

$\mathrm{RP}$ is associated with high morbidity and mortality in patients with lung cancer treated with CCRT. Therefore, it is essential to identify methods in the clinical setting to avoid the development of RP. The STRIPE score was developed using an individual patient meta-analysis dataset and includes clinical factors that may predict RP. However, the STRIPE score has not been validated in an independent cohort of patients. External validation is essential prior to implementing prediction models in clinical practice (16). Therefore, the current study validated the STRIPE score in an independent cohort and confirmed its discriminatory ability at the same level as in the original dataset (12).

The most important limitation of the STRIPE score was that a number of potential predictors of RP were not included in the model due to the lack of adequate data. As a result, the discriminatory ability of the model could be strengthened by including other relevant predictors that were excluded from the original model (12). By excluding the variables that are already included in the STRIPE score, current smoking was identified as a predictive factor against the development of RP. 
Table II. Bivariate analysis of potential risk factors for radiation-induced pneumonitis grade $\geq 2$.

\begin{tabular}{|c|c|c|c|}
\hline Variable & $\begin{array}{l}\text { Pneumonitis grade } 0-1 \\
\qquad(\mathrm{n}=55)\end{array}$ & $\begin{array}{l}\text { Pneumonitis grade } \geq 2 \\
\qquad(n=16)\end{array}$ & P-value \\
\hline Male gender ${ }^{\mathrm{a}}$ & $24(44)$ & $9(56)$ & 0.373 \\
\hline Age, years ${ }^{b}$ & $65(43-78)$ & $62.5(52-78)$ & 0.998 \\
\hline Current smoker ${ }^{\mathrm{a}}$ & $25(46)$ & $1(6)$ & 0.003 \\
\hline Presence of cardiac comorbidities ${ }^{\mathrm{a}}$ & $15(27)$ & $3(19)$ & 0.745 \\
\hline Presence of diabetes mellitus ${ }^{\mathrm{a}}$ & $6(11)$ & $2(13)$ & 1.000 \\
\hline Weight loss prior to diagnosis ${ }^{\mathrm{a}}$ & $15(37)$ & $7(44)$ & 0.618 \\
\hline Location (upper/lower) ${ }^{\mathrm{a}}$ & $22(44) / 28(56)$ & $7(47) / 8(53)$ & $0.874 / 0.949$ \\
\hline Location (right/left) ${ }^{\mathrm{a}}$ & $27(51) / 26(49)$ & $11(69) / 5(31)$ & $0.255 / 0.391$ \\
\hline Tumor size, $\mathrm{cm}^{\mathrm{b}}$ & $4.5(1.8-11.8)$ & $4.75(2.0-8.5)$ & 0.639 \\
\hline Tumor stage (IIIA/IIIB) ${ }^{\mathrm{a}}$ & $22(42) / 27(51)$ & $5(31) / 11(70)$ & $0.574 / 0.255$ \\
\hline Squamous histology ${ }^{a}$ & $20(39)$ & $6(43)$ & 0.805 \\
\hline Performance status $2^{\mathrm{a}}$ & $10(18)$ & $1(6)$ & 0.436 \\
\hline RT dose, $\mathrm{Gy}^{\mathrm{b}}$ & $60(46-68)$ & $60(50-68)$ & 0.217 \\
\hline Paclitaxel chemotherapy during $\mathrm{RT}^{\mathrm{a}}$ & $48(87)$ & $14(88)$ & 0.529 \\
\hline Cortisone during CRT & $10(19)$ & $5(31)$ & 0.308 \\
\hline MLD, Gy ${ }^{b}$ & $14(1.4-28)$ & $17.5(12-26)$ & 0.008 \\
\hline $\mathrm{V} 20, \%^{\mathrm{b}}$ & $23(0-44)$ & $31(19-50)$ & 0.004 \\
\hline PTV, $\mathrm{cm}^{\mathrm{b}}$ & $499(157-2,202)$ & $474(214-1,304)$ & 0.786 \\
\hline Intermediate risk STRIPE score ${ }^{\mathrm{a}}$ & $22(40)$ & $12(75)$ & 0.014 \\
\hline
\end{tabular}

Data are presented as ${ }^{\mathrm{a}} \mathrm{n}(\%)$ or ${ }^{\mathrm{b}}$ median (range). RT, radiotherapy; CRT, chemoradiotherapy; MLD, mean lung dose; PTV, planning target volume.

Table III. Discriminatory power of STRIPE score with and without smoking in the patient cohort.

\begin{tabular}{lcccr}
\hline & \multicolumn{2}{c}{ Hosmer-Lemeshow test } & & \multicolumn{2}{c}{ AUC } \\
\cline { 2 - 5 } Variable & $\chi^{2}$ & P-value & AUC & $95 \%$ CI \\
\hline STRIPE score & 7.79 & 0.45 & 0.68 & $0.53-0.82$ \\
STRIPE + smoking & 6.72 & 0.57 & 0.72 & $0.57-0.87$ \\
\hline
\end{tabular}

AUC, area under the curve; CI, confidence interval.

This finding is in line with previous studies indicating that ongoing smoking may be a protective factor against developing RP (11). The biological rationale for this observation is not clear, however, there are a number proposed theories, such as the diminished inflammatory response among smokers (17) and the lower production of cytotoxic free radicals due to the CO-induced hypoxia (18). Notably, the addition of smoking status to the STRIPE score increased the discriminatory ability of the model in the present study. Although this difference did not reach statistical significance, predominantly due to the limited sample size, the results underscore the possibility of increasing the discriminatory power of the model by including other predictors that were unable to be included in the original analysis (12).

Prior to accepting the current results, certain limitations should be taken into account. First, the retrospective nature of the study is prone to biases and the small sample size may influence the power to reveal other potential predictive factors. Second, the discriminatory power of the STRIPE score could not be validated in the current cohort to distinguish between high risk and intermediate risk, as no patients in the cohort were classified as high risk. Instead, the discriminatory power of the STRIPE score was validated to distinguish between intermediate risk and low risk of developing RP. A number of additional factors were included in the present analyses compared with the original analysis of STRIPE score (12); however, data was not gathered on other potential predictors of RP, such as baseline pulmonary function, the presence of lung fibrosis on baseline $\mathrm{CT}$, low partial pressure of $\mathrm{O}_{2}$ prior to radiotherapy and various biological factors, including transforming growth factor- $\mathrm{B}_{1}$ levels $(19,20)$.

In conclusion, barring the aforementioned caveats, the present study is a step forward to a more personalized approach to determining the risk of RP in patients with NSCLC treated 
with CCRT, as the study optimized the STRIPE score model for easier use in the clinical setting. Further research is essential to optimize and improve the model by investigating other clinical, biological and dosimetric factors prospectively in larger multicentric cohorts.

\section{References}

1. Jemal A, Siegel R, Xu J and Ward E: Cancer statistics, 2010. CA Cancer J Clin 60: 277-300, 2010.

2. Vansteenkiste J, De Ruysscher D, Eberhardt WE, Lim E, Senan S, Felip E and Peters S; ESMO Guidelines Working Group: Early and locally advanced non-small-cell lung cancer (NSCLC): ESMO clinical practice guidelines for diagnosis, treatment and follow-up. Ann Oncol 24 (Suppl 6): vi89-vi98, 2013.

3. Guida C, Maione P, Rossi A, Bareschino M, Schettino C, Barzaghi D, Elmo M and Gridelli C: Combined chemo-radiotherapy for locally advanced non-small cell lung cancer: Current status and future development. Crit Rev Oncol Hematol 68 222-232, 2008.

4. Aupérin A, Le Péchoux C, Rolland E, Curran WJ, Furuse K, Fournel P, Belderbos J, Clamon G, Ulutin HC, Paulus R, et al: Meta-analysis of concomitant versus sequential radiochemotherapy in locally advanced non-small-cell lung cancer. J Clin Oncol 28: 2181-2190, 2010.

5. O'Rourke N, Roqué I, Figuls M, Farré Bernadó N and Macbeth F: Concurrent chemoradiotherapy in non-small cell lung cancer. Cochrane Database Syst Rev: CD002140, 2010.

6. Byhardt RW, Scott C, Sause WT, Emami B, Komaki R, Fisher B, Lee JS and Lawton C: Response, toxicity, failure patterns, and survival in five radiation therapy oncology group (RTOG) trials of sequential and/or concurrent chemotherapy and radiotherapy for locally advanced non-small-cell carcinoma of the lung. Int J Radiat Oncol Biol Phys 42: 469-478, 1998.

7. Abid SH, Malhotra V and Perry MC: Radiation-induced and chemotherapy-induced pulmonary injury. Curr Opin Oncol 13: 242-248, 2001.

8. Rodrigues G, Lock M, D'Souza D, Yu E and Van Dyk J: Prediction of radiation pneumonitis by dose-volume histogram parameters in lung cancer-a systematic review. Radiother Oncol 71: 127-138, 2004.

9. Inoue A, Kunitoh H, Sekine I, Sumi M, Tokuuye K and Saijo N: Radiation pneumonitis in lung cancer patients: A retrospective study of risk factors and the long-term prognosis. Int J Radiat Oncol Biol Phys 49: 649-655, 2001.
10. Bradley JD, Hope A, El Naqa I, Apte A, Lindsay PE, Bosch W, Matthews J, Sause W, Graham MV and Deasy JO; RTOG: A nomogram to predict radiation pneumonitis, derived from a combined analysis of RTOG 9311 and Institutional data. Int J Radiat Oncol Biol Phys 69: 985-992, 2007.

11. Vogelius IR and Bentzen SM: A literature-based meta-analysis of clinical risk factors for development of radiation induced pneumonitis. Acta Oncol 51: 975-983, 2012.

12. Palma DA, Senan S, Tsujino K, Barriger RB, Rengan R, Moreno M, Bradley JD, Kim TH, Ramella S, Marks LB, et al: Predicting radiation pneumonitis after chemoradiation therapy for lung cancer: An international individual patient data meta-analysis. Int J Radiat Oncol Biol Phys 85: 444-450, 2013.

13. Goldstraw P, Crowley J, Chansky K, Giroux DJ, Groome PA, Rami-Porta R, Postmus PE, Rusch V and Sobin L; International Association for the Study of Lung Cancer International Staging Committee; Participating Institutions: The IASLC Lung Cancer Staging Project: proposals for the revision of the TNM stage groupings in the forthcoming (seventh) edition of the TNM Classification of malignant tumours. J Thorac Oncol 2: 706-714, 2007.

14. Sørensen JB, Klee M, Palshof T and Hansen HH: Performance status assessment in cancer patients. An inter-observer variability study. Br J Cancer 67: 773-775, 1993.

15. Cox JD, Stetz J and Pajak TF: Toxicity criteria of the Radiation Therapy Oncology Group (RTOG) and the European Organization for Research and Treatment of Cancer (EORTC). Int J Radiat Oncol Biol Phys 31: 1341-1346, 1995.

16. Bleeker SE, Moll HA, Steyerberg EW, Donders AR, Derksen-Lubsen G, Grobbee DE and Moons KG: External validation is necessary in prediction research: A clinical example. J Clin Epidemiol 56: 826-832, 2003.

17. Bjermer L, Franzén L, Littbrand B, Nilsson K, Angström T and Henriksson R: Effects of smoking and irradiated volume on inflammatory response in the lung of irradiated breast cancer patients evaluated with bronchoalveolar lavage. Cancer Res 50: 2027-2030, 1990.

18. Vaupel P, Thews $\mathrm{O}$ and Hoeckel M: Treatment resistance of solid tumors: Role of hypoxia and anemia. Med Oncol 18: 243-259, 2001.

19. Zhang XJ, Sun JG, Sun J, Ming H, Wang XX, Wu L and Chen ZT: Prediction of radiation pneumonitis in lung cancer patients: A systematic review. J Cancer Res Clin Oncol 138: 2103-2116, 2012.

20. Tsujino K, Hashimoto T, Shimada T, Yoden E, Fujii O, Ota Y, Satouchi M, Negoro S, Adachi S and Soejima T: Combined analysis of V20, VS5, pulmonary fibrosis score on baseline computed tomography, and patient age improves prediction of severe radiation pneumonitis after concurrent chemoradiotherapy for locally advanced non-small-cell lung cancer. J Thorac Oncol 9: 983-990, 2014. 